\title{
Influencia de la longitud de la biela en la eficiencia y biomecánica del pedaleo submáximo
}

\author{
V. RIVERO PALOMO ${ }^{1}$, V. FERRER-ROCA ${ }^{2}$, A. OGUETA-ALDAY ${ }^{1}$ \\ J.A. RODRÍGUEZ-MARROYO', J.GARCÍA-LÓPEZ ${ }^{1}$ \\ ${ }^{1}$ Facultad de Ciencias de la Actividad y del Deporte. Departamento de Educación Física y Deportiva. \\ Instituto de Biomedicina. Universidad de León \\ ${ }^{2}$ Centro de Alto Rendimiento (CAR), Sant Cugat del Vallés, Barcelona, España
}

\begin{abstract}
Resumen
El principal objetivo de este estudio es analizar el efecto de pequeños cambios en la longitud de la biela en el gasto energético y la técnica de pedaleo submáximo. Participaron 12 ciclistas de fondo en carretera bien entrenados, que realizaron pruebas de pedaleo a tres potencias con cadencia estable, utilizando tres longitudes de biela (en orden aleatorio). Sus bicicletas fueron exactamente reproducidas en un ergómetro que medía el torque ejercido sobre la biela izquierda y derecha de forma independiente. Simultáneamente se registró cinemática $2 D$ de la pierna derecha y el gasto energético al pedalear (eficiencia gruesa). Los resultados muestran que los cambios en la longitud de la biela no tuvieron efecto en la eficiencia gruesa, pero sí en las variables biomecánicas. Una biela de mayor longitud provocó una pérdida de eficiencia mecánica y un aumento en la flexión y el rango de movimiento de la cadera y la rodilla, sin cambios en el tobillo. Aunque estos cambios no fueron lo suficientemente importantes para alterar la eficiencia gruesa, sí que podrían tener implicaciones negativas a largo plazo (peor técnica de pedaleo y riesgo de lesión). En conclusión, el efecto de pequeños cambios en la longitud de la biela, que serían asumibles por ciclistas de competición, producen alteraciones en la biomecánica de pedaleo, pero no en su eficiencia gruesa. Futuros estudios deben abordar los efectos a largo plazo de estos cambios.
\end{abstract}

Palabras clave: Ciclismo; biomecánica; biela; cinética; cinemática; eficiencia gruesa.

\begin{abstract}
The main purpose of this study was to analyze the effects of small changes in crank length on gross efficiency and pedaling technique during submaximal cycling. Twelve well-trained road cyclists participated. They pedaled at three power outputs and steady cadence with three crank lengths (in a randomized order). Their bicycles were exactly reproduced on an ergometer where crank torque of the left and right legs were independently registered. $2 D$ kinematic of the right leg and energy cost (gross efficiency) were recorded. The results showed that changes in crank length had no effect on the gross efficiency, but had effect on the biomechanical variables. A longer crank caused a decreased in mechanical efficiency and an increase in the flexion and range of movement of the hip and the knee, without changes in the ankle. These alterations were not significant enough to alter the gross efficiency, but they could have negative long-term implications (worse pedaling technique and possible injuries). In conclusion, the small changes in crank arm length which are feasible for competitive cyclists affected cycling biomechanics, but not gross efficiency. Future studies should study long-term effects of these changes.
\end{abstract}

Keywords: Cycling, biomechanics, crank arm length, kinetics, kinematics, gross efficiency.

Correspondencia:

J. García-López

FCAFD. Universidad de León. C/ Campus de Vegazana S/N. 24071 - León, España

Email: juan.garcia@unileon.es

Teléfono: +34 987293018

Fax: +34 987293008 


\section{Introducción}

El rendimiento en ciclismo depende de varios factores fisiológicos, ligados al entrenamiento y biomecánicos $[17,18]$. La influencia de factores biomecánicos como la aerodinámica [23], las medidas de la bicicleta $[19,20]$ o los sistemas de pedaleo no circulares [43] en el rendimiento han sido demostrados. Sin embargo, la influencia de otros factores como la longitud de la biela son todavía una incógnita [33]. Mientras algunos estudios han descrito que esta variable afecta al rendimiento $[28,29,34,52]$, otros afirman lo contrario $[3,4,34,39,50]$. La mayoría de ellos han sido realizados durante esfuerzos supramáximos, con transferencia directa al ciclismo de pista, mientras que sólo unos pocos se han llevado a cabo durante esfuerzos submáximos, propios del ciclismo de ruta $[45,53]$. Esta división conceptual es necesaria, porque muchos de los análisis de optimización en ciclismo se han realizado para maximizar la producción de potencia a corto plazo, y no para minimizar el gasto de energía [26]. Así, es posible que una determinada configuración (ej. plato no circular) sea efectiva en esfuerzos supramáximos y no en esfuerzos submáximos [43], y viceversa.

Hasta donde llega nuestro conocimiento, siete estudios experimentales han analizado el efecto de la longitud de la biela en la producción de potencia (esfuerzos supramáximos). Tres de ellos encontraron diferencias, indicando que la mayor potencia se producía con bielas de longitud media (entre 145-180 mm), frente a bielas de longitudes extremas $(<140 \mathrm{y}>200 \mathrm{~mm})[28,35,52]$. Otros tres estudios no observaron diferencias al comparar bielas de 135 y $170 \mathrm{~mm}$ en niños [34], de 120 y $220 \mathrm{~mm}$ [50] y entre 150 y $190 \mathrm{~mm}$ [4] en ciclistas entrenados. Otro estudio fue realizado con pequeñas variaciones en la longitud de la biela $(170,172.5$ y $175 \mathrm{~mm})$ en mujeres practicantes de mountain bike, y aunque no se obtuvieron mejoras en la potencia, sí que observó un menor tiempo hasta la potencia máxima con bielas de menor longitud [33]. Los últimos hallazgos parecen coincidir en que la longitud de biela no afecta a la máxima producción de potencia en ciclismo $[4,33,50]$, aunque se ha destacado la necesidad de realizar trabajos con pequeñas variaciones en esta variable, de forma que sean modificaciones asumibles por los ciclistas experimentados [33].

La influencia de la longitud de la biela en el gasto de energía pedaleando (esfuerzos submáximos) ha sido investigada por cuatro estudios experimentales $[3,29,36,39]$. Uno de ellos analizó el efecto de utilizar bielas de 165,170 y $175 \mathrm{~mm}$, tras un período de entrenamiento ( 2 semanas y $>450$ $\mathrm{km})$, en 6 ciclistas experimentados. No se observaron diferencias entre las 3 longitudes de biela, ni al compararlas con la longitud de biela que habitualmente utilizaban los ciclistas [39]. Se obtuvieron los mismos resultados al analizar el gasto energético pedaleando con bielas de 160, 180 y $200 \mathrm{~mm}$ [3] y de 145, 170 y $195 \mathrm{~mm}$ [36]. Sin embargo, otro estudio sí observó diferencias entre utilizar bielas de 100 a $200 \mathrm{~mm}$, cifrando una longitud óptima de 140,150 y $160 \mathrm{~mm}$ para niños de 6,8 y 10 años [29]. Ninguno de los estudios mencionados tuvo en cuenta los cambios en la técnica de pedaleo (patrón cinemático y cinético). Desde un punto de vista biomecánico, se ha reconocido que existe discrepancia entre la longitud de biela utilizada en la práctica de ciclismo en ruta y la longitud óptima derivada de un modelo de optimización [27]. Esto pone de relieve la necesidad de realizar estudios experimentales de pedaleo submáximo, variando la longitud de la biela sobre los márgenes habitualmente utilizados por los ciclistas.

La mayoría de los estudios analizan grandes cambios en la longitud de la biela ( $>25 \mathrm{~mm}$ ), y sólo unos pocos realizan pequeños cambios (entre 2.5 y $5 \mathrm{~mm}$ ) que son asumibles por ciclistas entrenados [27,33,39]. Además, sería necesario considerar otras posibles implicaciones del cambio de la longitud de la biela, como su efecto en la técnica (cinemática y cinética) de pedaleo [27,52]. Por lo tanto, el principal objetivo de este estudio es analizar el efecto de pequeños cambios en la longitud de la biela $( \pm 5 \mathrm{~mm})$ en el gasto energético y la técnica de pedaleo submáximo. Como objetivo secundario también se analizará el efecto de la potencia de pedaleo en las variables mencionadas.

\section{Materiales y métodos}

\section{Sujetos}

Participaron 12 ciclistas de fondo en carretera bien entrenados (Tabla 1). Como criterios de inclusión se establecieron que llevaran compitiendo al menos 2 años en ciclismo, y que hubieran realizado más de $3000 \mathrm{~km}$ de entrenamiento en el momento de la evaluación. Todos ellos participaron voluntariamente y ninguno informó de algún problema médico en el momento del estudio. Se les informó de los procedimientos, métodos, beneficios y posibles riesgos involucrados en el estudio, y se obtuvo el consentimiento por escrito antes de iniciarlo. El protocolo de evaluación fue aprobado por el Comité de Ética de la Universidad y fue 
diseñado de acuerdo con las consignas de la Conferencia de Helsinki sobre investigación humana.

\section{Procedimientos}

Todos los ciclistas fueron evaluados al inicio de la temporada (febrero-mayo). El protocolo de evaluación se llevó a cabo en una sesión de un día en condiciones ambientales similares $\left(20-25^{\circ} \mathrm{C}\right.$, $60-65 \%$ de humedad relativa). Se citó a los ciclistas en el laboratorio ( $800 \mathrm{~m}$ de altitud), debiendo traer sus bicicletas, y respetando un período de 48 horas sin un entrenamiento duro. En primer lugar, se midieron las características antropométricas de los ciclistas y de las bicicletas (bielas incluidas). Estas medidas fueron trasladadas, junto con los pedales automáticos, a un cicloergómetro de freno electromagnético que permitía variar la longitud de las bielas (Lode Excalibur Sport, Lode BV, Groningen, Países Bajos). A continuación, los ciclistas realizaron un periodo de calentamiento de 10 minutos a una potencia de $100 \mathrm{~W}$, con un descanso de 5 minutos antes de empezar la prueba.

En segundo lugar, los ciclistas realizaron las pruebas de pedaleo submáximo con diferentes longitudes de biela, donde simultáneamente se llevaron a cabo los análisis biomecánico (cinemático y cinético) y fisiológico (gasto energético). Las pruebas consistieron en tres series de tres repeticiones de pedaleo submáximo. Las series se realizaron a tres potencias diferentes $(150,200 \mathrm{y}$ $250 \mathrm{~W}$ ), y dentro de cada una de ellas se utilizaron tres longitudes de biela (normal, corta y larga). La biela normal era la longitud de biela que habitualmente utilizaba el ciclista, mientras que la biela corta y larga diferían de aquélla en $5 \mathrm{~mm}$ (ej. 170,165 y $175 \mathrm{~mm}$, respectivamente). El orden de realización de las repeticiones con cada biela fue aleatorio para evitar posibles efectos de fatiga, del aprendizaje o de la oscilación del gasto de energía [32]. Cada repetición tenía una duración de $6 \mathrm{~min}$, con un descanso de 5 min entre repeticiones y 10 min entre series. El período de recuperación fue usado para cambiar la altura del sillín, de forma que se mantenía constante la distancia entre la parte alta del sillín y el eje del pedal en su punto más lejano [4]. La altura del manillar se ajustó en cada modificación de la altura del sillín para mantener la diferencia de alturas sillín-manillar, y las manos se colocaron en la parte superior del freno, para eliminar el impacto del coste metabólico de la modificación de la posición del tronco de los ciclistas [24]. Los ciclistas podían beber agua libremente después de cada serie, para evitar la deshidratación.
Las potencias de pedaleo de 150,200 y $250 \mathrm{~W}$ fueron seleccionadas por ser representativas del esfuerzo realizado en una etapa ciclista [53], y por permitir que el cociente respiratorio fuera menor de 1.00 en todos los ciclistas estudiados [43], indicando que no había contribución anaeróbica significativa durante el pedaleo. Aunque la mayor parte de los ciclistas finalizaron la prueba intentando completar una serie de $300 \mathrm{~W}$ con las tres bielas, la mitad $(\mathrm{n}=6)$ obtuvieron un cociente respiratorio mayor de 1.00 o no fueron capaces de completarla, motivo por el cual decidimos no incluir esta serie en el análisis de los datos. Los ciclistas recibieron información continua sobre su cadencia y se les pidió mantenerla constante a $90 \mathrm{rpm}$ para evitar cualquier posible influencia de la cadencia en las variables mecánicas de pedaleo [40]. La cadencia seleccionada es representativa de la cadencia de pedaleo sentado durante etapas llanas [44, 53].

\section{Medidas antropométricas y de la bicicleta}

Una cinta métrica (Holtain LTD; Crymych, UK) y un antropómetro (Harpenden, CMS instruments, London, UK) fueron utilizados para medir tanto la bicicleta como las dimensiones antropométricas de los ciclistas (Figura 1). Todas las mediciones fueron realizas por el mismo investigador. La talla, altura trocantérea y longitud de la entrepierna fueron registradas de acuerdo con estudios previos $[19,20]$. A continuación, se registraron las mediciones de la bicicleta: altura del sillín, retroceso del sillín, longitud de la biela, diferencia de alturas sillín-manillar y largura sillín-manillar [30]. La altura del sillín relativa (porcentaje) se calculó dividiendo la altura del sillín entre longitud de entrepierna [19].

\section{Análisis cinético}

La potencia de pedaleo fue fijada en el cicloergómetro de freno electromagnético, que ha sido validado y utilizado en estudios previos $[31,42]$. Este permitió la medición del torque ejercido sobre la biela izquierda y derecha de forma independiente cada $2^{\circ}$ de una revolución completa [14]. Antes de empezar el estudio, se llevó a cabo un procedimiento de calibración dinámica (Calibrator 2000, Lode BV, Groninger, Países Bajos), y se realizó un ajuste de cero antes de cada serie. Se registraron todas las pedaladas durante los $6 \mathrm{minu}-$ tos de cada repetición (software LEM, Lode BV, Groninger, Países Bajos). Para el análisis cinético se seleccionó la media de $\sim 360$ pedaladas completas entre el minuto uno y cinco, y se calculó 
el promedio de los valores de las bielas derecha e izquierda (Figura 2). Las siguientes variables mecánicas se obtuvieron directamente del software: frecuencia de pedaleo, torque máximo y torque mínimo. Además los datos de torque-tiempo y la longitud de la biela se exportaron a formato ASCI para calcular el resto de las variables mecánicas: impulso positivo, impulso negativo y eficiencia mecánica $(E M=$ impulso positivo $\times 100 \times \mid$ impulso positivo + impulso negativo|-1).

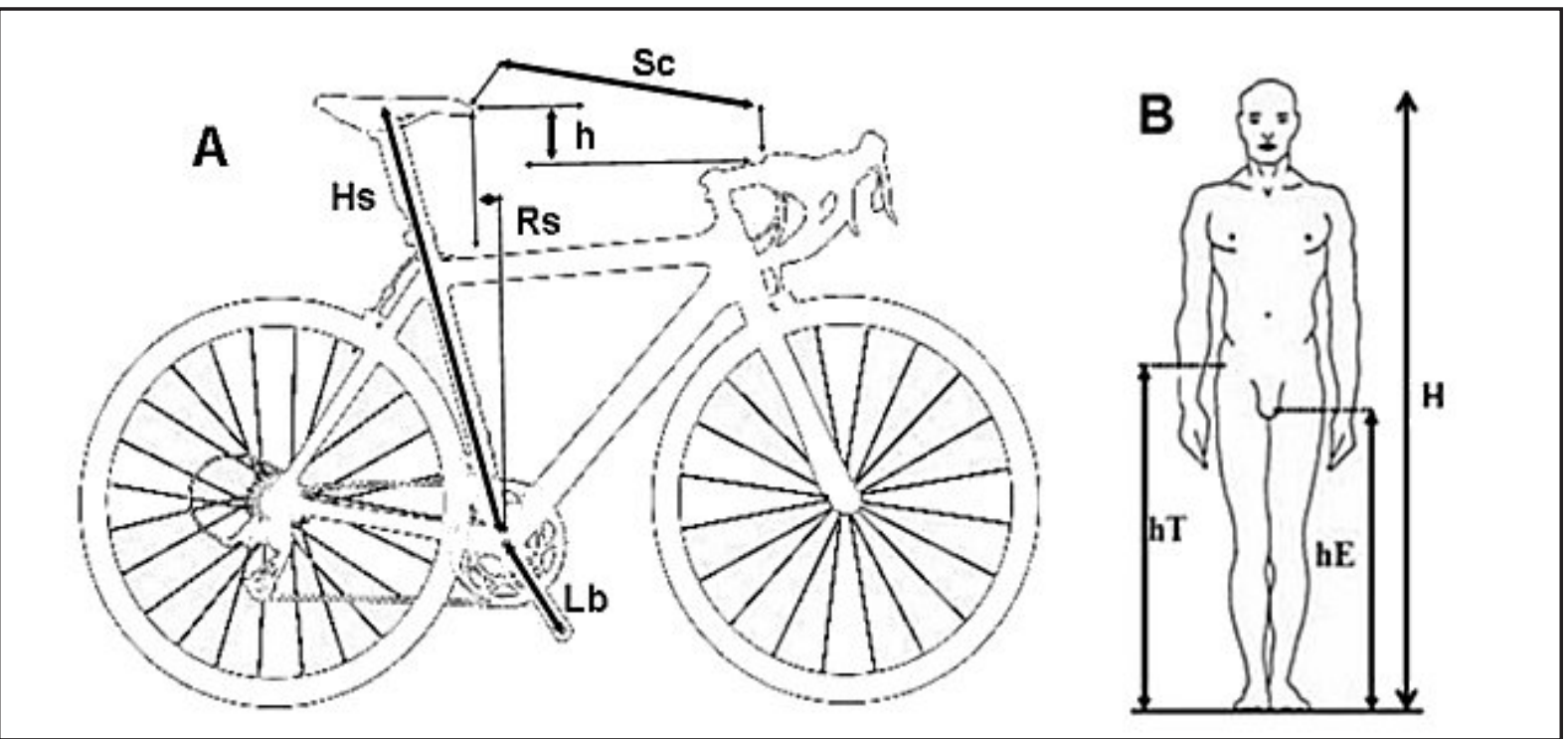

Figura 1. A: Medidas básicas que configuran la bicicleta: longitud de la biela (Lb), altura del sillín $(\mathrm{Hs})$, retroceso del sillín (Rs), largura sillín-manillar $(\mathrm{Sc})$ y diferencia de alturas entre el sillín y el manillar (h); B: Medidas antropométricas del ciclista: talla $(\mathrm{H})$, alturas trocantérea (hT) y de la entrepierna $(\mathrm{hE})$.

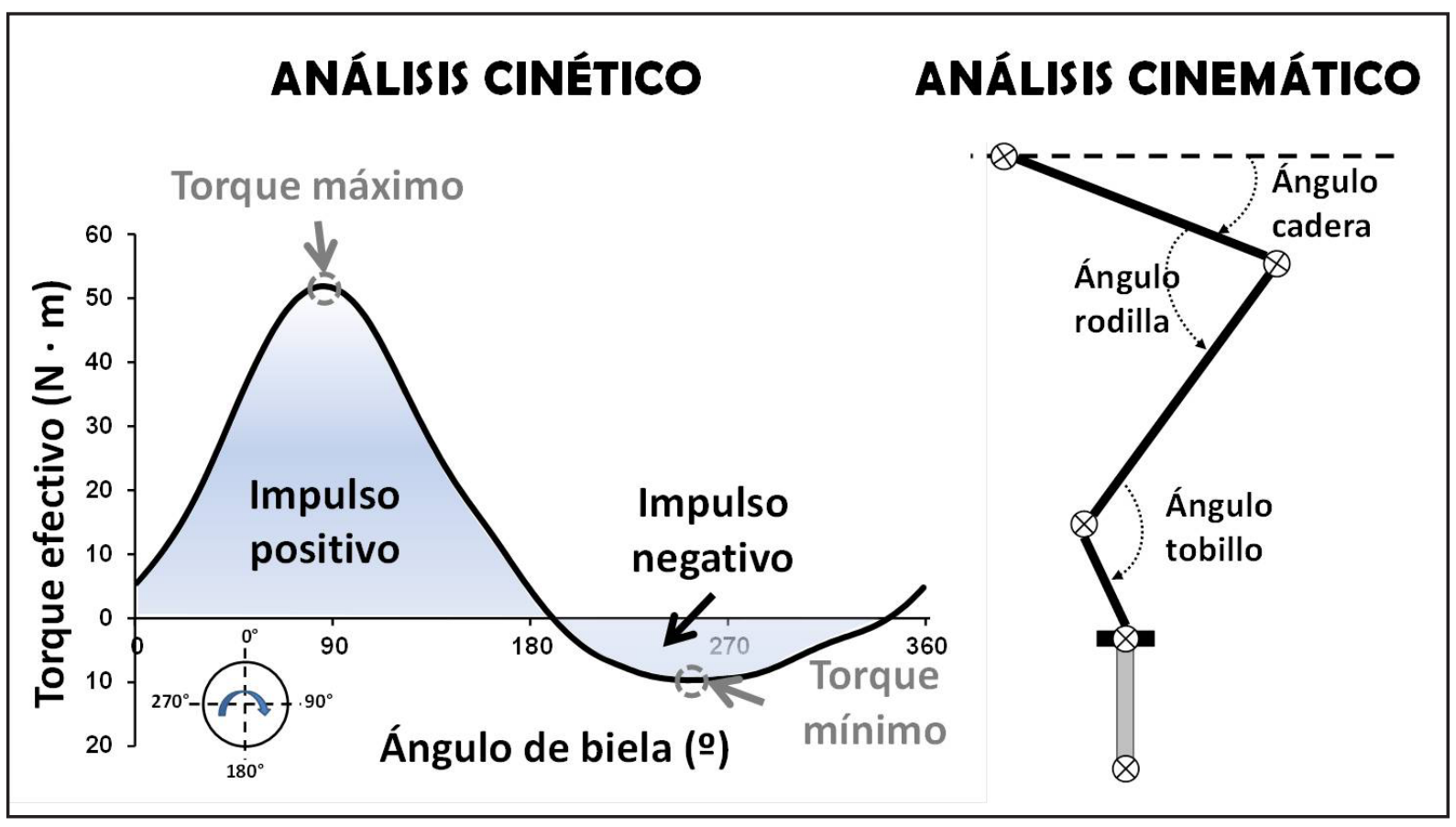

Figura 2. Variables biomecánicas analizadas durante el pedaleo. Análisis cinético: perfil de torqueángulo de una pedalada completa y las principales variables seleccionadas para el análisis. Análisis cinemático: representación esquemática de la ubicación de los marcadores reflectantes y la definición de los ángulos. 


\section{Análisis cinemático}

Se realizó el análisis cinemático del lado derecho de los ciclistas suponiendo simetría del movimiento entre los lados izquierdo y derecho [24]. Se pegaron en la piel de los ciclistas tres marcadores reflectantes de $10 \mathrm{~mm}$ de diámetro (trocánter mayor, epicóndilo lateral del fémur y maléolo lateral) y uno en la bicicleta (eje de rotación del pedal) $[6,19]$. Se fijó una cámara de video digital de alta velocidad (Sony Handycam HDR-HC7, Sony Inc, Europa, $200 \mathrm{~Hz}$ y $720 \times 576$ píxeles) y un foco a $4 \mathrm{~m}$ de distancia desde el plano sagital, donde se colocó un cuadro de calibración $(1.00 \times 1.20$ $\mathrm{m})$. El seguimiento automático, el procesamiento $\mathrm{y}$ análisis de datos fueron realizados mediante un software específico (Kinescan-IBV, versión 2001, Instituto de Biomecánica de Valencia, Valencia, España) [23]. Se analizaron seis pedaladas completas en los minutos 3 y 5 de cada prueba, utilizando la media como valor representativo. Se determinaron los ángulos de la cadera, la rodilla y el tobillo en el plano sagital, de acuerdo con convenciones previas $[5,6,7]$. Los valores de posición angular se expresaron como flexión (ángulo mínimo) y extensión (ángulo máximo). También se determinó el rango de movimiento de cada articulación (ROM), o diferencia ente la extensión y la flexión.

\section{Análisis del gasto energético}

El consumo de oxígeno y el cociente respiratorio registrados respiración a respiración (Medisoft Ergocard, Medisoft Group, Sorinnes, Belgium), así como la frecuencia cardiaca registrada cada 5 segundos (Polar Team, Polar Electro Oy, Kempele, Finland), fueron monitorizados de forma continua en todas las repeticiones, tomando la media de los últimos 3 minutos de cada repetición como valor representativo [25]. La eficiencia gruesa (EG) se calculó como la relación entre el trabajo mecánico externo realizado (Potencia x 0.01433 ) y la energía consumida o gastada ( $\mathrm{kcal}$ por litro de $\mathrm{O}_{2}$ $\mathrm{x} \mathrm{VO}_{2}$ ), de acuerdo con las ecuaciones publicadas recientemente [25]. Para calcular la energía consumida se tuvo en cuenta el cociente respiratorio registrado durante la prueba [43].

\section{Análisis estadístico}

Los resultados son expresados como media \pm DS, calculando además el intervalo de confianza al 95\% (IC95\%) de algunas de las variables analizadas. El software SPSS+ V.17.0 fue utilizado para el análisis estadístico (SPSS, Inc., Chicago, IL, USA). El test de Kolmogorov-Smirnov fue aplicado para asegurar la distribución normal de todas las variables analizadas. Un análisis de la varianza (ANOVA) de dos vías con medidas repetidas fue utilizado para analizar el efecto de la longitud de la biela y la potencia de pedaleo en las variables biomecánicas (cinemáticas y cinéticas) y fisiológicas (gasto energético). La prueba posthoc de Newman-Keuls fue utilizada para establecer diferencias entre las medias. Valores de $\mathrm{p}<$ 0.05 fueron considerados como estadísticamente significativos.

\section{Resultados}

La Tabla 1 muestra las principales características de los ciclistas que participaron en el estudio, así como las dimensiones de sus bicicletas. La longitud media de las bielas representó un $20.0 \pm 1.0 \%$ de la altura de la entrepierna (IC95\% $=19.4-20.7 \%)$. La altura relativa del sillín fue el $108.0 \pm 2.8 \%$ de la altura de la entrepierna (IC95\% $=106.2-109.8 \%$ ).

La Tabla 2 muestra que no se observó ningún efecto de la biela en la cadencia, frecuencia cardiaca y eficiencia gruesa ( $p>0.05)$. Se observó un efecto de la potencia en la frecuencia cardiaca $(\mathrm{F}=$ 249 y $\mathrm{p}<0.001)$ y la eficiencia gruesa $(\mathrm{F}=35.4$ y $\mathrm{p}<0.001)$. No hay efecto biela $\mathrm{x}$ potencia en ninguna de las variables analizadas $(\mathrm{p}>0.05)$.

La Tabla 3 muestra que la longitud de la biela tuvo un efecto significativo en el torque máximo $(\mathrm{F}=29.1$ y $\mathrm{p}<0.001)$, torque mínimo $(\mathrm{F}=$ 16.3 y $\mathrm{p}<0.001)$ y eficiencia mecánica $(\mathrm{F}=41.2$ y $\mathrm{p}<0.001)$. La potencia de pedaleo tuvo un efecto significativo en el torque máximo $(\mathrm{F}=198.5 \mathrm{y}$ $\mathrm{p}<0.001)$, torque mínimo $(\mathrm{F}=27.4$ y $\mathrm{p}<0.001)$ y eficiencia mecánica $(\mathrm{F}=273.5 \mathrm{y} \mathrm{p}<0.001)$. No hay efecto biela $\mathrm{x}$ potencia en ninguna de las variables analizadas $(\mathrm{p}>0.05)$.

La Tabla 4 muestra que una biela más larga aumentó la flexión de la cadera (entre 1.0 y $2.5^{\circ}, \mathrm{F}=$ 120.7 y $\mathrm{p}<0.001$ ) y de la rodilla (entre 1.6 y $3.4^{\circ}$, $\mathrm{F}=784.8$ y $\mathrm{p}<0.001)$, sin modificar los valores de extensión ( $\mathrm{p}>0.05)$, y consecuentemente aumentó el rango de movimiento de la cadera (entre $0.9 \mathrm{y}$ $2.2^{\circ}, \mathrm{F}=114.2$ y $\mathrm{p}<0.001$ ) y la rodilla (entre $1.3 \mathrm{y}$ $3.0^{\circ}, \mathrm{F}=245.7$ y $\mathrm{p}<0.001$ ). La potencia de pedaleo aumentó la extensión de la cadera (entre 1.1 y $1.9^{\circ}$, $\mathrm{F}=20.1$ y $\mathrm{p}<0.001$ ) y de la rodilla (entre 1.8 y $3.3^{\circ}$, $\mathrm{F}=28.3$ y $\mathrm{p}<0.001$ ), disminuyendo en menor medida la flexión de la cadera (entre 0.3 y $1.6^{\circ}, \mathrm{F}=9.9$ y $\mathrm{p}<0.01$ ) y de la rodilla (entre 0.5 y $1.6^{\circ}, \mathrm{F}=3.9$ y $\mathrm{p}<0.05$; respectivamente). Por lo tanto, la potencia de pedaleo aumentó el rango de movimiento en la 
cadera (entre 0.3 y $0.9^{\circ}, \mathrm{F}=4.8$ y $\mathrm{p}<0.05$ ) y la rodilla (entre 1.4 y $2.5^{\circ}, \mathrm{F}=12.1$ y $\mathrm{p}<0.001$, respectivamente). La extensión de tobillo no disminuyó de forma significativa $(\mathrm{p}>0.05)$, mientras que su flexión aumentó conforme aumentaba la potencia de pedaleo (entre 0.6 y $3.1^{\circ}, F=4.6$ y $\mathrm{p}<0.05$ ), sin cambios en su rango de movimiento ( $\mathrm{p}>0.05)$. No hay efecto biela $\mathrm{x}$ potencia en ninguna de las variables analizadas $(\mathrm{p}>0.05)$.

\begin{tabular}{|c|c|c|c|}
\hline & & Media \pm DE & Rango \\
\hline \multirow{7}{*}{ CICLISTAS } & Edad (años) & $20.8 \pm 2.8$ & $18.0-27.0$ \\
\hline & Peso (kg) & $68.5 \pm 6.6$ & $57.0-79.1$ \\
\hline & Talla (cm) & $176.9 \pm 6.4$ & $166.5-182.4$ \\
\hline & Altura trocantérea $(\mathrm{cm})$ & $90.3 \pm 3.7$ & $84.0-96.5$ \\
\hline & Altura entrepiena $(\mathrm{cm})$ & $86.7 \pm 4.5$ & $78.5-95.7$ \\
\hline & Experiencia ciclista (años) & $8.1 \pm 3.4$ & $3.0-11.0$ \\
\hline & Volumen de entrenamiento $(\mathrm{km})$ & $4063 \pm 1595$ & $3000-8000$ \\
\hline \multirow[t]{6}{*}{ BICICLETAS } & Altura del sillín (cm) & $76.1 \pm 3.3$ & $69.5-81.5$ \\
\hline & Altura del sillín (\%) & $108.0 \pm 2.8$ & $103.4-113.7$ \\
\hline & Retroceso del sillín $(\mathrm{cm})$ & $7.5 \pm 1.5$ & $4.7-10.2$ \\
\hline & Longitud de la biela (mm) & $173.3 \pm 1.2$ & $172.5-175.0$ \\
\hline & Largo sillín-manillar (cm) & $55.9 \pm 1.9$ & $52.2-58.5$ \\
\hline & Diferencia de alturas $(\mathrm{cm})$ & $9.0 \pm 1.8$ & $6.5-12.0$ \\
\hline
\end{tabular}

Tabla 1. Media \pm DS de las características de los ciclistas y las bicicletas que participaron en el estudio.

\begin{tabular}{|c|c|c|c|c|}
\hline & & Biela normal & Biela corta & Biela larga \\
\hline \multirow{3}{*}{$150 \mathrm{~W}$} & Cadencia (rpm) & $91.3 \pm 0.7$ & $91.4 \pm 0.6$ & $91.3 \pm 0.6$ \\
\hline & Frecuencia cardíaca (ppm) & $128 \pm 10+\ddagger$ & $129 \pm 10 \dagger \ddagger$ & $128 \pm 12+\ddagger$ \\
\hline & Eficiencia gruesa (\%) & $18.4 \pm 1.9+\ddagger$ & $18.6 \pm 1.3+\ddagger$ & $18.7 \pm 2.3+\ddagger$ \\
\hline \multirow{3}{*}{$200 \mathrm{~W}$} & Cadencia (rpm) & $91.3 \pm 0.7$ & $91.2 \pm 0.9$ & $91.4 \pm 0.8$ \\
\hline & Frecuencia cardíaca (ppm) & $144 \pm 13+$ & $143 \pm 13+$ & $145 \pm 14 \dagger$ \\
\hline & Eficiencia gruesa (\%) & $20.4 \pm 2.0+$ & $20.5 \pm 1.8+$ & $20.5 \pm 2.5+$ \\
\hline \multirow{3}{*}{$250 \mathrm{~W}$} & Cadencia (rpm) & $91.4 \pm 1.0$ & $91.4 \pm 0.7$ & $91.4 \pm 1.0$ \\
\hline & Frecuencia cardíaca (ppm) & $162 \pm 13$ & $161 \pm 15$ & $162 \pm 14$ \\
\hline & Eficiencia gruesa (\%) & $21.5 \pm 1.7$ & $22.0 \pm 1.9$ & $21.6 \pm 1.8$ \\
\hline
\end{tabular}

Tabla 2. Media \pm DS de las variables fisiológicas analizadas a las distintas potencias de pedaleo $(150,200$ y $250 \mathrm{~W})$ con las diferentes longitudes de biela (normal, corta y larga). 


\section{Discusión}

El principal hallazgo de este estudio fue que pequeños cambios en la longitud de la biela durante el pedaleo submáximo no tuvieron efecto en el gasto metabólico, pero sí en la biomecánica del pedaleo (cinemática y cinética). Una biela de mayor longitud provocó una pérdida de eficiencia mecánica, debida al mayor torque negativo durante la fase de recobro del ciclo de pedalada. Además, provocó un ligero aumento de la flexión y el rango de movimiento en la cadera y la rodilla, sin cambios en el tobillo. Los cambios cinéticos no tienen por qué manifestarse en el mismo sentido que los metabólicos, y los cambios cinemáticos no fueron lo suficientemente importantes como para tener repercusiones metabólicas. Sin embargo, estos cambios podrían tener relevancia a largo plazo.

La Tabla 1 muestra que las características antropométricas (peso y talla) de los ciclistas eran similares a las descritas en estudios previos para ciclistas amateurs [19,22], y ciclistas con un elevado nivel de experiencia en ciclismo (8.1 \pm 3.4 años). Respecto a la configuración de las bicicletas que traían los ciclistas, la altura relativa de su sillín $(108.0 \pm 2.8 \%)$ estaba dentro del rango recomendado para el pedaleo con rastrales (106-109\%), pero un poco baja comparada con el rango recomendado (108.6-110.4\%) para el pedaleo con pedales automáticos [19]. Es posible que los ciclistas amateurs tengan menor habilidad que los profesionales para ajustar la altura del sillín, adoptando posiciones relativamente más bajas [1]. La longitud media de las bielas concuerda con la longitud óptima recomendada en estudios previos (173.3 $\mathrm{mm}$ para una altura de la entrepierna de $86.7 \mathrm{~cm}$ ), y que equivale al $20 \%$ de la longitud de la entrepierna [34].

La longitud de la biela no tuvo influencia en ninguna de las variables fisiológicas analizadas (Tabla 2), por lo que puede afirmarse que el gasto metabólico fue similar. Destacar que la cadencia fue similar en todas las longitudes de biela y potencias analizadas, lo que resulta importante para comparar las variables fisiológicas y biomecánicas. La no influencia de la longitud de la biela en el gasto metabólico coincide con los resultados observados en la mayoría de los estudios experimentales previos sobre pedaleo submáximo, que no encontraron diferencias entre bielas de 160 a $200 \mathrm{~mm}$ [3], de 165 a $175 \mathrm{~mm}$ [39], y de 145 a $195 \mathrm{~mm}$ [36], con independencia de que las bielas fueran las que habitualmente utilizaban los ciclistas [39]. Sin embargo, debemos destacar que un estudio realizado en niños de 6 a 10 años apunta que longitudes de biela de $140 \mathrm{a} 160 \mathrm{~mm}$ son más económicas que longitudes de biela extremas de 100 ó $200 \mathrm{~mm}$ [29]. De la misma forma, dos comunicaciones presentadas a congresos obtienen resultados contradictorios, y mientras una obtiene que no hay diferencias significativas entre utilizar 7 longitudes de biela entre 165 y $180 \mathrm{~mm}$ [12], la otra sí que observa diferencias entre utilizar 6 longitudes de biela entre 150 y $200 \mathrm{~mm}$ [8]. A partir

\begin{tabular}{|c|c|c|c|c|}
\hline & & Biela normal & Biela corta & Biela larga \\
\hline \multirow{3}{*}{$150 \mathrm{~W}$} & Torque máximo $(\mathrm{N} \cdot \mathrm{m})$ & $37.6 \pm 4.5+\ddagger$ & $37.2 \pm 4.4^{* \dagger \ddagger}$ & $38.2 \pm 4.5^{\dagger}$ \\
\hline & Torque mínimo $(\mathrm{N} \cdot \mathrm{m})$ & $-13.9 \pm 3.4+\ddagger$ & $-13.3 \pm 3.0 *+\ddagger$ & $-14.3 \pm 3.2+\mp$ \\
\hline & Eficiencia mecánica (\%) & $76.3 \pm 3.7^{*}+\ddagger$ & $76.8 \pm 3.2 *+\ddagger$ & $75.7 \pm 3.5+\ddagger$ \\
\hline \multirow{3}{*}{$200 \mathrm{~W}$} & Torque máximo $(\mathrm{N} \cdot \mathrm{m})$ & $44.8 \pm 5.3 *+$ & $44.0 \pm 5.4 * \#$ & $45.7 \pm 5.4 \dagger$ \\
\hline & Torque mínimo $(\mathrm{N} \cdot \mathrm{m})$ & $-12.6 \pm 3.1 *+$ & $-12.0 \pm 2.9 * \#+$ & $-13.1 \pm 2.7 \dagger$ \\
\hline & Eficiencia mecánica (\%) & $80.9 \pm 4.0 \dagger$ & $81.4 \pm 4.2 * \dagger$ & $80.5 \pm 3.7 \dagger$ \\
\hline \multirow{3}{*}{$250 \mathrm{~W}$} & Torque máximo $(\mathrm{N} \cdot \mathrm{m})$ & $50.4 \pm 5.5^{*}$ & $49.8 \pm 5.5^{*}$ & $52.1 \pm 5.6$ \\
\hline & Torque mínimo $(\mathrm{N} \cdot \mathrm{m})$ & $-10.5 \pm 2.3 *$ & $-9.2 \pm 3.3 * \#$ & $-11.4 \pm 2.5$ \\
\hline & Eficiencia mecánica (\%) & $85.8 \pm 3.3 *$ & $86.5 \pm 3.6 * \#$ & $84.6 \pm 3.5$ \\
\hline
\end{tabular}

Tabla 3. Media \pm DS de las variables cinéticas analizadas a las distintas potencias de pedaleo (150, 200 y $250 \mathrm{~W}$ ) con las diferentes longitudes de biela (normal, corta y larga). 
de los resultados obtenidos en el presente estudio, la mayor evidencia científica se posiciona en la no influencia de la longitud de la biela en el gasto metabólico del pedaleo.

De forma lógica, la potencia de pedaleo aumentó el gasto metabólico, aumentando la frecuencia cardiaca. También aumentó la eficiencia grue- sa desde $18.4-18.7 \%$ a $150 \mathrm{~W}$ hasta $21.5-22.0 \%$ a $250 \mathrm{~W}$, lo que concuerda con estudios previos realizados en ciclistas amateurs hasta los $\sim 250 \mathrm{~W}$ [11]. Estudios más recientes han mostrado que la eficiencia gruesa aumenta con el incremento de la potencia de pedaleo hasta el $50 \%$ de la potencia máxima, manteniéndose estable desde este punto

\begin{tabular}{|c|c|c|c|c|c|}
\hline \multirow{10}{*}{$150 \mathrm{~W}$} & \multirow{4}{*}{ CADERA } & & \multirow{2}{*}{$\frac{\text { Biela normal }}{62.1 \pm 2.8+}$} & \multirow{2}{*}{$\frac{\text { Biela corta }}{62.0 \pm 3.1+}$} & \multirow{2}{*}{$\frac{\text { Biela larga }}{62.2 \pm 2.6+}$} \\
\hline & & Extension (으) & & & \\
\hline & & Flexion (ㅇ) & $17.4 \pm 2.2 *+$ & $18.1 \pm 2.2 * \#+\ddagger$ & $16.3 \pm 2.5+$ \\
\hline & & ROM () & $44.7 \pm 2.6 * \ddagger$ & $43.9 \pm 2.7 * \#$ & $45.9 \pm 2.7$ \\
\hline & & Extension (우) & $143.0 \pm 3.6+\mp$ & $143.1 \pm 4.2+\ddagger$ & $\begin{array}{c}143.2 \pm 3.5 \dagger \\
\ddagger\end{array}$ \\
\hline & RODILLA & Flexion (으) & $69.2 \pm 2.2 *+$ & $70.5 \pm 2.3 * \#+$ & $67.6 \pm 2.2$ \\
\hline & & ROM () & $73.8 \pm 3.5 *+\ddagger$ & $72.6 \pm 3.9 * \# \dagger \ddagger$ & $75.5 \pm 3.6+\ddagger$ \\
\hline & & Extension (ㅇ) & $136.8 \pm 5.4$ & $136.8 \pm 5.8$ & $137.0 \pm 4.9$ \\
\hline & TOBILLO & Flexion (ㅇ) & $118.4 \pm 7.0 \dagger$ & $118.9 \pm 7.0 \dagger$ & $118.3 \pm 7.8$ \\
\hline & & ROM & $18.4 \pm 4.0$ & $18.0 \pm 4.1+$ & $18.8 \pm 5.3$ \\
\hline \multirow{9}{*}{$200 \mathrm{~W}$} & \multirow{3}{*}{ CADERA } & Extension (우 & $63.6 \pm 3.2+$ & $63.6 \pm 2.8+$ & $63.3 \pm 3.3+\ddagger$ \\
\hline & & Flexion (ㅇ) & $17.9 \pm 2.4 *+$ & $19.1 \pm 2.4 * \# \dagger$ & $16.6 \pm 1.8+$ \\
\hline & & ROM (o) & $45.6 \pm 2.8 *$ & $44.5 \pm 2.3 * \#$ & $46.7 \pm 2.6$ \\
\hline & \multirow{3}{*}{ RODILLA } & Extension (우 & $145.2 \pm 4.2 \dagger$ & $145.4 \pm 4.1 \dagger$ & $145.0 \pm 4.6+$ \\
\hline & & Flexion (ㅇ) & $69.7 \pm 2.1 *$ & $71.4 \pm 2.3 * \#$ & $68.0 \pm 2.0$ \\
\hline & & ROM () & $75.5 \pm 3.9 *$ & $74.0 \pm 3.8 * \# \dagger$ & $77.0 \pm 4.2 \dagger$ \\
\hline & \multirow{3}{*}{ TOBILLO } & Extension (우 & $135.6 \pm 6.5$ & $135.6 \pm 5.6$ & $135.7 \pm 5.8$ \\
\hline & & Flexion (ㅇ) & $117.8 \pm 8.5$ & $117.9 \pm 7.6+$ & $117.7 \pm 6.9$ \\
\hline & & ROM & $17.9 \pm 4.4$ & $17.7 \pm 4.0$ & $18.0 \pm 3.6$ \\
\hline \multirow{9}{*}{$250 \mathrm{~W}$} & \multirow{3}{*}{ CADERA } & Extension (ㅇ) & $64.0 \pm 3.3$ & $63.9 \pm 3.1$ & $64.0 \pm 3.0$ \\
\hline & & Flexion (ㅇ) & $18.6 \pm 2.4 *$ & $19.7 \pm 2.2 * \#$ & $17.6 \pm 2.3$ \\
\hline & & ROM (o) & $45.4 \pm 2.6 *$ & $44.2 \pm 2.7^{*} \#$ & $46.3 \pm 2.5$ \\
\hline & \multirow{3}{*}{ RODILLA } & Extension (우) & $146.3 \pm 4.8$ & $146.2 \pm 4.7$ & $145.9 \pm 4.2$ \\
\hline & & Flexion (ㅇ) & $70.0 \pm 2.2 *$ & $71.3 \pm 2.1 * \#$ & $68.2 \pm 2.3$ \\
\hline & & ROM (ㅇ) & $76.3 \pm 4.5^{*}$ & $74.9 \pm 4.4 * \#$ & $77.6 \pm 4.5$ \\
\hline & \multirow{3}{*}{ TOBILLO } & Extension (ㅇ) & $135.1 \pm 5.1$ & $135.1 \pm 5.0$ & $134.9 \pm 5.4$ \\
\hline & & Flexion (으) & $115.8 \pm 6.8$ & $115.8 \pm 6.8$ & $115.7 \pm 7.5$ \\
\hline & & ROM & $19.3 \pm 4.6$ & $19.3 \pm 3.8$ & $19.2 \pm 4.8$ \\
\hline $\begin{array}{l}\text { Diferenc } \\
\text { Potencia }\end{array}$ & cativas & 0.05): Biel & $\begin{array}{l}\text { W) } \\
\text { Wpec }\end{array}$ & & ta vs Biela \\
\hline
\end{tabular}

Tabla 4. Media \pm DS de las variables cinemáticas analizadas a las distintas potencias de pedaleo $(150,200$ y $250 \mathrm{~W})$ con las diferentes longitudes de biela (normal, corta y larga). 
en adelante [13]. Desafortunadamente el presente trabajo no incluyó una valoración de la potencia máxima de los ciclistas. Sin embargo, diez de los doce ciclistas fueron capaces de realizar una prueba de pedaleo a $300 \mathrm{~W}$ con las tres longitudes de biela, obteniendo valores similares ( $\mathrm{p}>0.05)$ a los mostrados a $250 \mathrm{~W}(21.8-22.3 \%$ vs $21.5-22.0 \%$, respectivamente). Estos datos no fueron finalmente incluidos en el análisis, porque dos ciclistas no fueron capaces de finalizar la prueba y otros cuatro obtuvieron un cociente respiratorio mayor de 1.00, quedando la muestra disponible sólo en 6 ciclistas.

La longitud de la biela tuvo efecto en las variables biomecánicas del pedaleo (Tablas 3 y 4). Se observó que para pedalear entre 150 y $250 \mathrm{~W}$ los ciclistas necesitaban un mayor torque máximo (entre 1.0 y $2.3 \mathrm{~N} \cdot \mathrm{m}$ ) y perdían eficiencia mecánica (entre 0.9 y $1.9 \%$ ) con las bielas de mayor longitud, porque el torque mínimo aumentaba (entre 1.0 y $2.2 \mathrm{~N} \cdot \mathrm{m}$ ) (Tabla 3 ). Esto pudo ser debido al hecho de que entre los $\sim 210-330^{\circ}$ del ciclo de pedaleo el torque de biela es negativo [31], y una biela más larga facilita un mayor torque negativo, que debe ser compensado con un mayor torque positivo, para mantener la misma potencia de pedaleo. En cierta medida, estos resultados contradicen la creencia de que una mayor longitud de biela requiere de una menor aplicación de fuerza en el pedal $[16,51]$. La ganancia de eficiencia mecánica con la biela más corta fue similar a la mostrada tras un periodo de entrenamiento de $\sim 9000 \mathrm{~km}$ en ciclistas profesionales [22], y la mitad que las diferencias observadas entre ciclistas profesionales y amateurs [22]. Los cambios en la cinética del pedaleo no tuvieron impacto en la eficiencia gruesa del pedaleo, de la misma forma que se ha descrito que un pedaleo más eficiente desde el punto de vista mecánico no tiene por qué serlo desde el punto de vista fisiológico $[31,38,49]$. Sin embargo, estos cambios biomecánicos pueden tener interesantes implicaciones en pruebas de mayor duración (ej. etapas ciclistas) o a largo plazo [49], cuestión que debe investigarse en un futuro.

De otra parte, los valores de extensión en las articulaciones de cadera, rodilla y tobillo fueron similares en las distintas longitudes de biela, porque la altura del sillín fue modificada (Tabla 4) con el objetivo de mantener constante la distancia sillín-pedal [51]. Advertimos de esta circunstancia, porque algunos estudios previos no modificaron la altura del sillín al cambiar la longitud de la biela [37]. Dicho esto, las bielas de mayor longitud aumentaron los valores de flexión y el ROM en la cadera (entre 0.9 y $2.5^{\circ}$ ) y la rodilla (entre
1.3 y $3.4^{\circ}$ ), sin cambios en el tobillo. Estos resultados son completamente coincidentes con los observados en estudios previos sobre pedaleo supramáximo [52]. Sin embargo, las modificaciones cinemáticas no tuvieron impacto en el gasto metabólico, posiblemente porque son muy pequeñas en comparación con las referidas en otros estudios que cambiaron la longitud de la biela (4-14 ${ }^{\circ}$ cada $\sim 35 \mathrm{~mm}$ de biela) o la altura del sillín (3-4 ${ }^{\circ}$ cada $2 \%$ de cambio en la altura del sillín) $[20,52]$. Sin embargo, una biela más larga provocaba que el valor absoluto de flexión de cadera fuese inferior a los $20^{\circ}$ y el de rodilla inferior a los $70^{\circ}$ que han sido descritos en estudios previos $[9,19,21,41]$. La máxima flexión de rodilla y cadera acontece alrededor del punto muerto superior del pedaleo, donde la actividad de los músculos del cuádriceps es máxima en los primeros $90^{\circ}$ del ciclo de pedaleo [15]. Esto, a largo plazo, podría suponer una sobresolicitación de los músculos extensores de la rodilla en la fase inicial del ciclo de pedaleo. De hecho, se ha asociado el dolor en la parte anterior de la rodilla con la utilización de bielas demasiado largas $[2,48]$. Futuros estudios deberían evaluar el efecto a largo plazo del uso de bielas de excesiva longitud.

El aumento de la potencia también afectó a las variables biomecánicas del pedaleo (Tablas 3 y 4). El torque máximo se incrementó, mientras que el torque mínimo disminuyó, causando por tanto un aumento de la eficiencia mecánica (Tabla 3). Estos resultados están en la línea de estudios anteriores, en los que el principal efecto de aumentar la potencia de pedaleo a cadencia constante fue un aumento en la fuerza máxima aplicada al pedal $[46,47]$ y una disminución del peso que recaía sobre el mismo [22]. La disminución del torque mínimo con el aumento de la potencia de pedaleo se considera una "estrategia del ciclista para mejorar la aplicación efectiva de fuerza para reducir la necesidad de hacer fuerza con la pierna propulsora" [46]. Este efecto ha sido descrito recientemente como una estrategia para retrasar la fatiga en la musculatura extensora de la rodilla, implicando en mayor medida la musculatura flexora de la pierna contralateral [49]. Los cambios más importantes se encontraron a $250 \mathrm{~W}$, posiblemente debido a que los ciclistas destacaron su mejor potencial [47], como así indica su mejor valor de eficiencia gruesa.

Las variables cinemáticas también se vieron afectadas por el incremento de la potencia de pedaleo, aumentando la extensión de cadera y rodilla en mayor medida que disminuía su flexión, 
y aumentando la flexión de tobillo (Tabla 4). El aumento en la extensión de cadera y la flexión de tobillo está en la línea de los resultados obtenidos en trabajos previos [5]. Una de las principales respuestas al aumento de la potencia de pedaleo es aumentar la actividad del músculo tibial anterior [15], que es el principal agonista de la flexión de tobillo. La mayor extensión y menor flexión en la cadera ha sido descrita como una diferencia entre ciclistas de diferentes niveles competitivos [21], lo que podría ser una adaptación al pedaleo a mayores potencias de los ciclistas profesionales respecto a los ciclistas amateurs [22]. Esto conllevaría también una mayor flexión de tobillo y, posiblemente, un mayor rango de movimiento a largo plazo, que ha sido descrito como un factor diferencial entre ciclistas de diferente nivel [22]. Nótese que en el presente estudio el rango de movimiento del tobillo de los ciclistas amateurs analizados siempre es inferior a $20^{\circ}$, mientras que estudios previos con ciclistas de más nivel muestran valores de $23.5-24.5^{\circ}$ [21]. Futuros trabajos son necesarios para clarificar la posible influencia de la cinemática del tobillo en el rendimiento en ciclismo, cuestión que fue sólo discutida hace bastante tiempo [10].

La principal limitación del estudio ha sido no haber realizado una prueba de esfuerzo máxima antes de los ensayos con bielas, de forma que se hubiera conocido el $\mathrm{VO}_{2 \max }$ y la intensidad relativa $\left(\% \mathrm{VO}_{2 \max }\right)$ de los ciclistas pedaleando a las distintas potencias $(150,200$ y $250 \mathrm{~W})$. Sin embargo, consideramos importante haber utilizado las mismas intensidades absolutas (ej. $200 \mathrm{~W}$ ) en vez de las mismas intensidades relativas (ej. 70\% del $\mathrm{VO}_{2 \max }$ ó $3 \mathrm{~W} \cdot \mathrm{kg}^{-1}$ ) con el objetivo de poder interpretar el comportamiento de las variables biomecánicas, que deben ser analizadas a distintas potencias absolutas de pedaleo [22,32]. Otra limitación del estudio ha sido no contar con un mayor número de sujetos, de forma que se hubieran podido establecer correlaciones entre las variables fisiológicas y biomecánicas analizadas. Igualmente, con sujetos de diferente nivel competitivo, podría haberse analizado la posible influencia de este factor, junto con la longitud de biela, en las variables fisiológicas y biomecánicas analizadas. Futuros trabajos deberían resolver las limitaciones mencionadas.

\section{Conclusiones}

Este es el primer estudio que analiza conjuntamente los efectos agudos, fisiológicos y bio- mecánicos, de introducir pequeños cambios en la longitud de la biela $( \pm 5 \mathrm{~mm})$ durante el pedaleo submáximo. Estos cambios serían asumibles por ciclistas de competición en ruta. Respecto a las bielas que habitualmente utilizan los ciclistas, estos cambios no producen modificaciones en la eficiencia metabólica del pedaleo, lo que coincide con la mayoría de los estudios previos. Sin embargo, se producen alteraciones en la biomecánica de la pedalada conforme aumenta la longitud de la biela, siendo menos eficiente mecánicamente porque se necesita una mayor fuerza para pedalear a la misma potencia, que es debida a un mayor peso dejado en los pedales durante la fase de recobro. Además, una biela de mayor longitud aumenta la flexión de las articulaciones de cadera y rodilla, lo cual podría tener implicaciones negativas a largo plazo, relacionadas con la solicitación de los músculos que participan en el pedaleo. Futuros estudios deben abordar los efectos a largo plazo de estos pequeños cambios en la longitud de la biela.

\section{Agradecimientos}

A todos los ciclistas que voluntaria y desinteresadamente aceptaron formar parte de este trabajo, y sin los cuales no hubiera podido llevarse a cabo. A Raúl Pernía, José Alberto Caveda, Alejandro Bartol y Ana Ogueta, por su inestimable ayuda durante la realización de la fase experimental del estudio. Gracias también al Consejo Superior de Deportes (Gobierno de España) por financiar los estudios de nuestro laboratorio sobre biomecánica del ciclismo (proyectos 12/UPB10/07 y 18/ UPB10/08), y al Gobierno Vasco por contribuir con la beca predoctoral de investigación de Dña. Ana Ogueta.

\section{Bibliografía}

1. Alvarez G. Análisis y optimización biomecánica de la técnica de pedaleo ciclista. San Sebastián. Tesis Doctoral. Universidad de Navarra, 1995.

2. Asplund C, Pierre PS. Knee pain and bicycling: fitting concepts for clinicians. Phys Sportsmed 2004;32:23-30.

3. Astrand PO. Study of bicycle modifications using a motor driven treadmill-bicycle ergometer. Arbeitsphysiologie 1953;15:23-32.

4. Barratt PR, Korff T, Elmer SJ, Martin JC. Effect of crank length on joint specific power during maximal cycling. Med Sci Sports Exerc 2011;43:1689-1697.

5. Bini RR, Diefenthaeler F. Kinetics and kinematics analysis of incremental cycling to exhaustion. 
Sports Biomech 2010;9:223-235.

6. Bini RR, Diefenthaeler F, Mota CB. Fatigue effects on the coordinative pattern during cycling: kinetics and kinematics evaluation. J Electromyogr Kinesiol 2010a;20:102-107.

7. Bini RR, Tamborindeguy AC, Mota CB. Effects of saddle height, pedaling cadence, and workload on joint kinetics and kinematics during cycling. $\mathrm{J}$ Sport Rehabil 2010b;19:301-314.

8. Carmichael JKS, Loumis JL, Hodgson L. The effect of crank length on oxygen consumption and heartrate when cycling at a constant power output. Med Sci Sports Exerc 1982;14:162.

9. Carpes FP, Dagnese F, Mota CB, Stefanyshyn DJ. Cycling with noncircular chainring system changes the three-dimensional kinematics of the lower limbs. Sports Biomech 2009;8:275-283.

10. Cavanagh PR, Sanderson DJ. The Biomechanics of cycling: studies of the pedalling mechanics of elite pursuit riders. In: Burke ER, editor. Science of Cycling. Champaign, Illinois: Human Kinetics, 1986. p. 91-122.

11. Chavarren J, Calbet JA. Cycling efficiency and pedalling frequency in road cyclists. Eur J Appl Physiol 1999;80:555-563.

12. Conrad DP. Bicycle crank arm length and oxygen consumption in trained cyclists. Med Sci Sports Exerc 1983;15:111-112.

13. De Koning JJ, Noordhof DA, Lucia A, Foster C. Factors affecting gross efficiency in cycling. Int J Sports Med 2012;33:880-885.

14. Dorel S, Couturier A, Hug F. Influence of different racing positions on mechanical and electromyographic patterns during pedalling. Scand J Med Sci Sports 2009; 19:44-54.

15. Enders H, Maurer C, Baltich J, Nigg BM. Taskoriented control of muscle coordination during cycling. Med Sci Sports Exerc 2013;45:22982305.

16. Faria IE. Energy expenditure, aerodynamics and medical problems in cycling. An update. Sports Med 1992;14:43-63.

17. Faria EK, Parker DL, Faria IE. The science of cycling. Physiology and Training - Part 1. Sports Med 2005a;35:285-312.

18. Faria EK, Parker DL, Faria IE. The science of cycling. Factors affecting performance - Part 2. Sports Med 2005b;35:313-337.

19. Ferrer-Roca V, Roig A, Galilea P, García-López $\mathbf{J}$. Influence of saddle height on lower limb kinematics in well-trained cyclists. Static versus dynamic evaluation in bike fitting. J Strength Cond Res 2012;26:3025-3029.

20. Ferrer-Roca V, Bescós R, Roig A, Galilea P, Valero O, García-López J. Acute effects of small changes in bicycle saddle height on gross efficiency and lower limb kinematics. J Strength Cond Res 2014;28:784-791.

21. García-López J, Díez-Leal S, Rodríguez-Marroyo JA, Larrazabal J, De Galdeano IG, Villa JG. Eficiencia mecánica de pedaleo en ciclistas de diferente nivel competitivo. Biomecánica 2009;17:9-20.

22. García-López J, Ogueta-Alday A, Díez-Leal S, Larrazabal J, Rodríguez-Marroyo JA. Differences in pedalling technique between road cyclists of different competitive levels. J Sports Sci (en revisión).

23. García-López J, Rodríguez-Marroyo JA, Juneau CE, Peleteiro J, Martínez AC, Villa JG. Reference values and improvement of aerodynamic drag in professional cyclists. J Sports Sci 2008;26:277-286.

24. Heil DP, Derrick TR, Whittlesey S. The relationship between preferred and optimal positioning during submaximal cycle ergometry. Eur J Appl Physiol 1997;75:160-165.

25. Hopker J, Coleman D, Jobson SA, Passfield L. Inverse relationship between $\mathrm{VO} 2 \mathrm{max}$ and gross efficiency. Int J Sports Med 2012;33:789-794.

26. Hull ML, Williams M, Williams K, Kautz S. Physiological response to cycling with both circular and noncircular chainrings. Med Sci Sports Exerc 1992;24:1114-1122.

27. Hull ML, Gonzalez H. Bivariate optimization of pedalling rate and crank arm length in cycling. J Biomech 1988;21:839-849.

28. Inbar O, Dotan R, Trousil T, Dvir Z. The effect of bicycle crank-length variation upon power performance. Ergonomics 1983;26:1139-1146.

29. Klimt F, Voigt GB. Studies for the standardisations of the pedal frequency and the crank length at the work on the bicycle-ergometer in children between 6 and 10 years of age. Eur J Appl Physiol 1974;33:315-326.

30. Korff T, Fletcher G, Brown D, Romer LM. Effect of "Pose" cycling on efficiency and pedaling mechanics. Eur J Appl Physiol 2011;111:1177-1186.

31. Korff T, Romer LM, Mayhew I, Martin JC. Effect of pedaling technique on mechanical effectiveness and efficiency in cyclists. Med Sci Sports Exerc 2007;39:991-995.

32. Leirdal S; Ettema G. Pedaling technique and energy cost in cycling. Med Sci Sports Exerc 2011;43:701-705.

33. Macdermid PW, Edwards AM. Influence of crank length on cycle ergometry performance of well-trained female cross-country mountain bike athletes. Eur J Appl Physiol 2010;108:177-182. 
Martin JC, Malina RM, Spirduso WW. Effects

34. os crank length on maximal cycling power and optimal pedaling rate of boys aged 8-11 years. Eur J Appl Physiol 2002;86:215-217.

Martin JC, Spirduso WW. Determinants of

35. maximal cycling power: crank length, pedaling rate and pedal speed. Eur J Appl Physiol 2001;84:413-418.

McDaniel J, Durstine JL, Hand GA, Martin

36. JC. Determinants of metabolic cost during submaximal cycling. J Appl Physiol 2002;93:823828.

Mileva K, Turner D. Neuromuscular and bio-

37. mechanical coupling in human cycling. Adaptations to changes in crank length. Exp Brain Res 2003;152:393-403.

Mornieux G, Stapelfeldt B, Gollhofer A, Belli

38. A. Effects of pedal type and pull-up action during cycling. Int J Sports Med 2008;29:817-822.

Morris DM, Londeree BR. The effects of bi-

39. cycle crank arm length on oxygen consumption. Can J Appl Physiol 1997;22:429-438.

Neptune RR, Herzog W. The association bet-

40. ween negative muscle work and pedaling rate. $\mathrm{J}$ Biomech 1999;32:1021-1026.

Price D, Donne B. Effect of variation in seat

41 tube angle at different seat heights on submaximal cycling performance in man. J Sports Sci 1997; 15:395-402.

Reiser M, Meyer T, Kindermann W, Daugs R.

42. Transferability of workload measurements between three different types of ergometer. Eur J Appl Physiol 2000;82:245-249.

Rodríguez-Marroyo JA, García-López J, Cha-

43. mari K, Córdova A, Hue O, Villa JG. The rotor pedaling system improves anaerobic but not aerobic cycling performance in professional cyclists. Eur J Appl Physiol 2009;106:87-94.

Rodríguez-Marroyo JA, García-Lopez J, Vi-

44. Ila JG, Cordova A. Adaptation of pedaling rate of professional cyclist in mountain passes. Eur J
Appl Physiol 2008;103:515-522.

Rodríguez-Marroyo JA, García-López J, Avila

45. C, Jiménez F, Cordova A, Villa JG. Intensity of exercise according to topography in professional cyclists. Med Sci Sports Exerc 2003;35:12091215.

Sanderson DJ. The influence of cadence and 46. power output on the biomechanics of force application during steady-rate cycling in competitive and recreational cyclists. J Sports Sci 1991;9:191203.

Sanderson DJ, Henning EM, Black AH. The

47. influence of cadence and power output on force application and in shoe pressure distribution during cycling by competitive and recreational cyclists. J Sports Sci 2000;18:173-181.

Silberman MR, Webner D, Collina S, Shi-

48. ple BJ. Road Bicycle Fit. Clin J Sport Med 2005; 15:271-276.

Theurel J, Crepin M, Foissac M, Temprado

49. JJ. Effects of different pedalling techniques on muscle fatigue and mechanical efficiency during prolonged cycling. Scand J Med Sci Sports 2012;22:714-721.

Tomas A, Ross EZ, Martin JC. Fatigue during

49. maximal sprint cycling: unique role of cumulative contraction cycles. Med Sci Sports Exerc 2010;42:1364-1369.

Too D. Biomechanics of cycling and factors

50. affecting performance. Sports Med 1990;10:286302.

Too D, Landwer GE. The effect of pedal crank

51. arm length on joint angle and power production in upright cycle ergometry. J Sports Sci 2000;18:153-161.

Vogt S, Schumacher YO, Roecker K, Dic-

52. khuth HH, Schoberer U, Schmid A, Heinrich L. Power Output during the Tour de France. Int J Sports Med 2007;28:756-761. 